\title{
Role of Pir1 in the construction of the Candida albicans cell wall
}

\author{
Ana I. Martínez, Luis Castillo, Ana Garcerá, María V. Elorza, \\ Eulogio Valentín and Rafael Sentandreu
}

Correspondence

Rafael Sentandreu

Rafael.sentandreu@uv.es

Received 7 April 2004

Revised 16 June 2004

Accepted 25 June 2004

\author{
Departament de Microbiología i Ecología, Facultat de Farmacia, Universitat de València, Vicent \\ Andrés Estelles s/n, 46100 Burjassot, València, Spain
}

\begin{abstract}
Searches in a Candida albicans database (http://genolist.pasteur.fr/CandidaDB/) identified two Individual Protein Files (IPF 15363 and 19968) whose deduced amino acid sequences showed $42 \%$ and $45 \%$ homology with Saccharomyces cerevisiae Pir4. The two DNA sequences are alleles of the same gene (CaPIR1) but IPF 19968 has a deletion of 117 bases. IPF 19968 encodes a putative polypeptide of 364 aa, which is highly O-glycosylated and has an $N$-mannosylated chain, four cysteine residues and seven repeats. Both alleles are expressed under different growth conditions and during wall construction by regenerating protoplasts. The heterozygous mutant cells are elongated, form clumps of several cells and are hypersensitive to drugs that affect cell wall assembly. CaPir1 was labelled with the V 5 epitope and found linked to the 1,3- $\beta$-glucan of the C. albicans wall and also by disulphide bridges when expressed in S. cerevisiae.
\end{abstract}

\section{INTRODUCTION}

The cell wall of Candida albicans plays essential roles in growth and development, and in interactions with mammalian cells and tissues during infection and colonization. Besides its primary protective role in shielding the cell against osmotic, chemical and biological harm, the wall of C. albicans is involved in many other functions, including morphogenesis, and some activities that may be termed 'social', such as morphological responses, antigenic expression, adhesion and cell-cell interaction (Calderone \& Fonzi, 2001; Berman \& Sudbery, 2002; Sundstrom, 2002). There are many data supporting the idea that temporal and spatial regulation of the synthesis and assembly of wall polymers are critical for the properties of the wall, which thus do not exclusively depend on their chemical composition, but also on the way that different polymers interact and assemble to give the final structure.

The cell wall is a composite of chitin fibrils immersed in an amorphous matrix made of $\beta$-glucans and mannoproteins. The three components are dispersed throughout the cell wall, although the mannoproteins are mostly concentrated on the outer surface (Sentandreu et al., 2001; Klis et al., 2002; Kapteyn et al., 1999b).

In C. albicans and Saccharomyces cerevisiae some wall proteins are released by hot SDS and the remaining ones are solubilized in the form of supramolecular highly polydisperse complexes, after the degradation of the structural

Abbreviations: ConA, concanavalin A; Endo-F, endoglycosidase F; IPF, individual protein file. skeleton by $\beta$-glucanases and chitinase (Marcilla et al., 1991, 1993). Three types of proteins that are released by $\beta$-glucanases and chitinase have been detected: glycosylphosphatidylinositol proteins (GPI-dependent wall proteins), Pir proteins (proteins with internal repeats) and other proteins. The proteins of the first group are linked to $1,3-\beta$ glucan through a 1,6- $\beta$-glucan connector whereas the proteins of the second group are highly $O$-glycosylated, and are attached to the 1,3- $\beta$-glucan in the cell wall by unknown alkali-labile bonds, suggesting that they are retained through an $\mathrm{O}$-glycosydic linkage (Mormeneo et al., 1995; Mrša \& Tanner, 1999; Kapteyn et al., 2000; Klis et al., 2001). The third type of proteins detected in the cell walls lack signal peptide and are probably exported by a putative, non-classical export pathway: enolase, aconitase, pyruvate kinase, phosphoglycerate mutase, methionine synthase and many others have been identified (Cleves et al., 1996; Eroles et al., 1997; Pardo et al., 1999, 2000).

Pir proteins seem to be also present in C. albicans and other fungi, as immunological and Southern and Northern experiments suggested the presence of a protein related to the heat-shock-inducible product of the HSP150/PIR2 gene of S. cerevisiae (Kandasamy et al., 2000; Kapteyn et al., 2000; Jaafar et al., 2003).

Kapteyn et al. (1999a) published evidence that the amount of Hsp150/Pir2 in the walls of 1,6- $\beta$-glucan-deficient mutants increases and suggested that this phenomenon is part of a general compensatory mechanism in response to cell wall weakening caused by low levels of $1,6-\beta$-glucan (Kapteyn et al., 1999a). 
This paper reports identification of a homologue of S. cerevisiae Pir4 by a BLAST search in a C. albicans genomic database (http://genolist.pasteur.fr/CandidaDB/). This new C. albicans Pir protein, CaPirl, has two alleles of different sizes. Both alleles seem to be expressed equally. After cloning, the $C$. albicans Pir protein was expressed in S. cerevisiae and overexpressed in C. albicans. The protein was labelled with V5 epitope and found to be linked by alkaline sensitive linkages to the wall.

\section{METHODS}

Micro-organisms and growth conditions. C. albicans, S. cerevisiae and Escherichia coli strains, and the plasmids used in this study, are listed in Tables 1 and 2, respectively. Strains were maintained on slants of YPD solid medium (1\% yeast extract, $2 \%$ peptone, $2 \%$ glucose, $2 \%$ agar). Inocula were prepared by transfer of loopfuls of cells from slants to YNB or YPD liquid medium, depending on the experiment, and the cells were incubated by shaking overnight at the appropriate temperature. YNB-maltose medium contained $2 \%$ maltose, $0 \cdot 167 \%$ YNB and $0.5 \%\left(\mathrm{NH}_{4}\right)_{2} \mathrm{SO}_{4}$. E. coli $\mathrm{DH} 5 \alpha$ was grown at $37^{\circ} \mathrm{C}$ in Luria-Bertani (LB) broth supplemented with ampicillin $\left(100 \mu \mathrm{g} \mathrm{ml}^{-1}\right)$ when appropriate. Agar was added to $1 \cdot 5 \%$ for LB plates.

Preparation of wall and soluble fractions. Yeast cells were collected by centrifugation $(3000 \mathrm{~g}, 10 \mathrm{~min})$, washed twice with chilled distilled water, then suspended in a small volume of $1 \mathrm{mM}$ PMSF and broken by vortexing with glass beads ( $5 \mathrm{mg}$ beads per $\mathrm{mg}$ cells); complete cell breakage was obtained. Cell breakage was assessed by examining the preparation with a phase-contrast microscope. The cell walls were sedimented $(3000 \mathrm{~g}, 10 \mathrm{~min})$ from the cell-free homogenate, washed four times with chilled distilled water, then boiled for $10 \mathrm{~min}$ (twice) with $2 \%$ SDS in distilled water, and finally washed a further six times with chilled $1 \mathrm{mM}$ PMSF in distilled water. Isolated walls were freeze-dried and stored at $-20{ }^{\circ} \mathrm{C}$ until further use.

Table 1. Strains

\begin{tabular}{|c|c|c|}
\hline Strain & Genotype & Source \\
\hline \multicolumn{3}{|l|}{ C. albicans } \\
\hline SC5314 & Wild-type & Gillum et al. (1984) \\
\hline CAI4/pADH-15 & 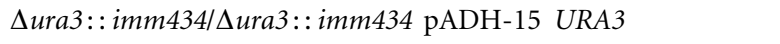 & This work \\
\hline CAI4/pADH-15-myc & $\Delta$ ura3:: imm434/Dura3::imm434 pADH-15-myc URA3 & This work \\
\hline CAPIR19 & $\triangle i p f 19968 /$ IPF15363: : URA3 & This work \\
\hline \multicolumn{3}{|l|}{ S. cerevisiae } \\
\hline BMA $64-1 \mathrm{~A}$ & 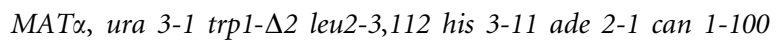 & EUROFAN \\
\hline $\operatorname{pir} 4 \Delta$ & $\begin{array}{l}\text { MATa ura 3-1 trp1- } \Delta 2 \text { leu2-3,112 his 3-11 ade 2-1 can 1-100 } \\
\text { pir4:: KanMX4 }\end{array}$ & Moukadiri et al. (1999) \\
\hline \multicolumn{3}{|l|}{ E. coli } \\
\hline $\mathrm{DH} 5 \alpha$ & $\begin{array}{l}\mathrm{F}^{-} \phi 80 \mathrm{~d} \text { lac } \Delta \mathrm{M} 15 \text { recA1 endA1 gyrA96 thi-1 hsdR17 }\left(\mathrm{r}_{\mathrm{k}}^{-} \mathrm{m}_{\mathrm{k}}^{-}\right) \\
\text {supE44 relA1 deoR } \Delta(\text { lacZYA-argF }) U 169\end{array}$ & Hanahan (1985) \\
\hline
\end{tabular}

Table 2. Plasmids

\begin{tabular}{|lll|}
\hline Plasmid & \multicolumn{1}{c|}{ Characteristics } & Reference \\
\hline pADH-pl & Contains the promoter of ADH1 of C. albicans, CaURA3, Amp ${ }^{\mathrm{R}}$ & Bertram et al. (1996) \\
Clp10-MAL2p & Contains the promoter of CaMAL2, CaURA3, Amp & Backen et al. $(2000)$ \\
p5921 & Contains the hisG-URA3-hisG cassette, CaURA3, Amp & Fonzi \& Irwin $(1993)$ \\
p15-1,2 & p5921 containing the 15-1,2 fragment & This work \\
p15-c & p15-1,2 containing the 15-3,4 fragment & This work \\
pADH-15 & pADH-pl containing the ORF of CaPIR1 & This work \\
pADH-15-myc & pADH-pl containing the ORF of CaPIR1 fused to the $c$-myc epitope & This work \\
pMAL-15-V5 & CIp10-MAL2p containing the ORF of CaPIR1 fused to the V5 epitope & This work \\
\hline
\end{tabular}


The cell walls (100 mg dry weight) were suspended in a solution of $0.01 \mathrm{M}$ ammonium acetate, $\mathrm{pH} 6 \cdot 3$, containing $2 \%(\mathrm{v} / \mathrm{v}) \quad \beta$ mercaptoethanol, shaken for $3 \mathrm{~h}$ at $28^{\circ} \mathrm{C}$ and then pelleted. The supernatant was concentrated by freeze-drying and dissolved in Laemmli solution (1970). The cell wall residue was then extracted again with $15 \mathrm{mM} \mathrm{NaOH}$ (Kapteyn et al., 1999a).

Protein gel electrophoresis and Western blot techniques. Proteins were separated by SDS-PAGE performed basically as described elsewhere (Laemmli, 1970) in $10 \%(w / v)$ acrylamide gels loaded with $20 \mu \mathrm{g}$ protein. The gels were stained with Coomassie brilliant blue or transferred onto Hybond-C nitrocellulose membranes as described by Towbin et al. (1979) and Burnette (1981), and immunodetected with a polyclonal antibody preparation against the material extracted by Zymolyase from C. albicans yeast cell walls (PAbL) (1:1000), or anti-c-myc $(1: 500)$ and anti-V5 (1:500) monoclonal antibodies (Invitrogen). Detection was carried out by the enhanced chemiluminiscent (ECL) method from Amersham Biosciences, following the manufacturer's instructions.

Mannoproteins were stained by the protocol described by Hawkes (1982) and modified by Millete \& Scott (1984). To remove Nglycosylated chains of mannoproteins, samples containing $15 \mu \mathrm{g}$ protein were treated with endoglycosidase F (Endo-F; Roche) following the manufacturer's instructions.

Protoplast preparation and regeneration. Preparation and regeneration of C. albicans CAI4 protoplasts were carried out as described by Elorza et al. (1983). Regeneration was performed for $30 \mathrm{~min}, 1 \mathrm{~h}, 2 \mathrm{~h}, 3 \mathrm{~h}$ and $5 \mathrm{~h}$. Regenerated protoplasts in each condition were recovered by centrifugation $(10 \mathrm{~min}, 2000 \mathrm{~g})$ and stored at $-20^{\circ} \mathrm{C}$.

Mycelium induction. For germ tube induction, cells were cultured in modified Lee's medium as described previously (Elorza et al., 1988).

Heat-shock assay. C. albicans CAI4 was grown overnight at $25^{\circ} \mathrm{C}$ in YNB medium at 200 r.p.m. One millilitre was inoculated into a $110 \mathrm{ml} \mathrm{YNB}$ medium preheated at $25^{\circ} \mathrm{C}$ and was grown at $25^{\circ} \mathrm{C}$ to $\mathrm{OD}_{600} 0 \cdot 6$. Two $50 \mathrm{ml}$ samples were taken. One was added to $50 \mathrm{ml}$ YNB medium preheated at $25^{\circ} \mathrm{C}$ and incubated at $25^{\circ} \mathrm{C}$ for $30 \mathrm{~min}$. The other was added to $50 \mathrm{ml} \mathrm{YNB}$ medium preheated at $53^{\circ} \mathrm{C}$ and incubated at $37^{\circ} \mathrm{C}$ for $30 \mathrm{~min}$. Cultures were harvested at room temperature by centrifugation $(2000 \mathrm{~g}, 5 \mathrm{~min})$ and stored frozen until RNA extraction.

Genetic constructions. To construct the heterozygous PIR1 mutants, part of the gene (522 bp) was replaced with a hisGURA3:: hisG cassette (Fonzi \& Irwin, 1993). This cassette was made by a two-step PCR amplification procedure. In the first step, an amplicon of $512 \mathrm{bp}$ was obtained from genomic DNA using primers 15-1 (TTCCGAGCTCGTTAGTATTGTTACTTTGTTAG) and 15-2 (TTCCGGTACCTTTGTTATCATTTCTAGTCAATG) containing

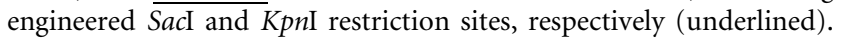
The amplicon obtained (15-1,2), which included the nucleotides -291 to 224, was digested with $S a c \mathrm{I}$ and $K p n \mathrm{I}$ and cloned into p5921 previously digested with the same enzymes, producing plasmid p15-1,2. In a second step, a fragment of 411 bp was obtained by using primers 15-3 (TTCCGTCGACTACTGCTGAAAATGTTGCTAAAGC) and 15-4 (TTCCCTGCAGTTTAACAGTTGACAAATTCAATG), which have SalI and PstI restriction sites, respectively (underlined). This new amplicon, 15-3,4, which included nucleotides 746 to 1185 , was digested with SalI and PstI and cloned into p15-1,2 digested with the same enzymes, producing plasmid p15-c.

Disruption of both alleles was attempted as described by Fonzi \& Irwin (1993). C. albicans CAI4 cells were transformed with $10 \mu \mathrm{g}$ of a SacIPstI fragment from p15-c. Cells were selected in YNB medium lacking uridine and integration was checked by PCR, RT-PCR and Southern blot analysis. Some of the heterozygous disruptants obtained from each allele $\left(P I R 1_{15363} /\right.$ pir $_{19968} \Delta$ and $\left.p i r_{15363} \Delta / P I R_{19968}\right)$ were plated on YNB medium containing uridine and 5-fluoro-orotic acid to select $\mathrm{Ura}^{-}$revertants produced as a result of recombination of the flanking his $G$ repeats (Boeke et al., 1984).

C. albicans CAI4/pADH-15 and S. cerevisiae pir $4 \Delta / \mathrm{pADH}-15$ strains with constitutive expression of CaPIR1 were obtained by transformation of S. cerevisiae pir4A mutant or C. albicans CAI4 with plasmid pADH-15. To construct this plasmid the CaPIRl coding region was PCR-amplified. Primers designed to introduce a BglII site at position -1 with respect to the first nucleotide of the coding region and an EcoRV site in the $3^{\prime}$ end were used. The primer sequences were $\mathrm{ADH}$ $155^{\prime}$ (TACTTGAGATCTATGAAGTACTCTACACTTGTTAG) and ADH 153' (GGGCCCGATATCTTAATAGTTGACAAACTC); they include BglII and EcoRV sites respectively (underlined). The PCR product was digested with $B g l I I$ and $E c o$ RV and ligated with the $12 \cdot 1 \mathrm{~kb}$ BglII-EcoRV fragment of pADH-pl (Bertram et al., 1996) to generate pADH-15.

To construct pADH-15-myc the coding region of CaPIR1 was amplified by PCR using the oligonucleotides ADH $155^{\prime}$ and ADH 15-myc 3' (CCTTGATATCTAAAGATCCTCTTCTGAGATGAGTTTTTGTTCACAGTTGACAAATTCAATGACAC) containing the $c$ - $m y c$ sequence (in bold), the stop codon TAA, an EcoRV restriction site (underlined) and $23 \mathrm{bp}$ of the $3^{\prime}$ part of the CaPIR1 gene sequence. The PCR product obtained using these primers produced a $1150 \mathrm{bp}$ band that was eluted and digested with $B g l I I$ and EcoRV, and ligated with the $12 \cdot 1 \mathrm{~kb}$ fragment of pADH-pl digested with the same enzymes. This new construct was called pADH-15-myc.

Plasmid pMAL-15-V5 was constructed to tag the CaPir1 protein with a V5 epitope. The CaPIR1 coding region was amplified with primers designed to introduce an EcoRV site at position - 1 with respect to the first nucleotide of the coding region and an MluI site at the $3^{\prime}$ end. The sequence corresponding to the V5 epitope was added at the $3^{\prime}$ end. The primer sequences used were $15-\mathrm{V} 55^{\prime}$ (TTAGGATATCATGAAGTATTCTACACTTGTTAGTATTGCTGC), which includes the EcoRV site (underlined), and 15-V5 3' (GACCACGCGTTTACGTAGAATCGAGACCGAGGAGAGGGTTAGGGATAGGCTTACCACAGTTGACAAATTCAATG), which contains a MluI site (underlined), the stop codon TAA and the V5 epitope sequence (in bold). The $1162 \mathrm{bp} \mathrm{PCR} \mathrm{product}$ was digested with EcoRV and MluI and ligated with a 5.68 kb EcoRV/ MluI Clp10-MAL2p fragment to generate the plasmid pMAL-15-V5. The identity of all PCR products was confirmed by cloning and sequencing.

Clp10-MAL2p (Backen et al., 2000) contains the inducible C. albicans $M A L 2$ promoter, the gene $C a R P 10$ and the C. albicans URA3 as marker.

pMAL-15-V5 was digested with $\mathrm{NcoI}$ and the linear $6.9 \mathrm{kDa}$ fragment was used to transform C. albicans CAI4. Integration was performed in the RP10 locus. Transformants were recovered in YNB medium and grown in YNB-maltose medium to induce tagged protein expression.

Transformation of C. albicans and S. cerevisiae. S. cerevisiae was transformed using the lithium acetate method as described by Ito et al. (1983) and modified by Gietz \& Sugino (1988). C. albicans was transformed using the protocol described by Gietz et al. (1992).

Phenotypic analysis of the pir1/PIR1 heterozygous strains. Morphological differences and alterations in growth rates were tested. Calcofluor white and Congo red sensitivity were tested by streaking cells onto YNB medium plates containing increasing concentrations of the two compounds as described Van der Vaart et al. (1995). Cells were grown in YNB liquid medium overnight and adjusted to $\mathrm{OD}_{600} 1$, serial decimal dilutions were done and drops 
of $3 \mu \mathrm{l}$ were placed onto the surface of YNB plates containing increasing concentrations of Calcofluor white $\left(0-150 \mu \mathrm{g} \mathrm{ml}^{-1}\right)$ and Congo red $\left(0-30 \mu \mathrm{g} \mathrm{ml}^{-1}\right)$. Plates were incubated at $28^{\circ} \mathrm{C}$ and monitored after 3 days.

RT-PCR analysis. Total RNA was prepared from C. albicans CAI4 after growth in different conditions by the method described by Langford \& Gallwitz (1983).

RNA was treated with RNase-free DNase I (Amersham Biosciences) to eliminate genomic DNA contamination. The first-strand cDNA synthesis reaction was catalysed by the SuperScript First Strand Synthesis System for RT-PCR (Invitrogen) and the primers used to amplify IPF 15363 and IPF 19968 were ADH $155^{\prime}$ and ADH 15 3'. PCR generated an amplicon of $1120 \mathrm{bp}$ from the first allele and another of 1003 bp for IPF 19968. In a parallel experiment and as an internal control the RT-PCR was performed using new primers that amplify CaRPSO (Baquero et al., 2001). This gene carries an intron, and genomic DNA gave a fragment of $870 \mathrm{bp}$ and cDNA one of $545 \mathrm{bp}$. The oligonucleotides used in this case were YST11B (TTTGACTTAACTCCAGAAGACGG) and YST12B (ACCTCTTAATCTCAAGACTTCTCTAGC). cDNA was quantified in a GeneQuant II spectrophotometer (Amersham Pharmacia Biotech) and all samples contained the same quantity of first-strand cDNA $(1 \mu \mathrm{g})$ for PCR amplification. In some experiments, several cycles of amplification were analysed and PCR products were run on $0 \cdot 8 \%$ agarose gels.

\section{RESULTS}

\section{Identification of putative Pir proteins in C. albicans}

$\mathrm{N}$-terminal sequencing of a protein extracted by $\beta$ mercaptoethanol from $S$. cerevisiae cell walls with an apparent mobility of $40 \mathrm{kDa}$ in SDS-PAGE gave the sequence DVITQIGDGQVQA. This sequence was $100 \%$ identical to a peptide contained in S. cerevisiae ORF YJL158c (Castillo et al., 2003). This ORF has been previously described by Moukadiri et al. (1999) and other researchers as Pir4/Ccw5p/Ccw1p/Scw8p/Cis3. In order to localize Pir4 homologues in C. albicans, isolated cell walls, previously extracted with hot SDS, were treated with $\beta$-mercaptoethanol and the released material fractionated by SDS-PAGE. No homologues to the $40 \mathrm{kDa}$ S. cerevisiae protein were detected, instead a polydisperse material, higher than $70 \mathrm{kDa}$, was found (data not shown). After this result, homology searches were carried out in silico. To find potential Pir4 homologues in C. albicans, a database of this organism (http://genolist.pasteur.fr./CandidaDB) was analysed using a BLAST approach. Two ORFs IPF (Individual Protein File) 15363 and IPF 19968, were identified as homologues to S. cerevisiae Pir4. The two ORFs showed homology of $42 \%$ and $45 \%$, respectively, with the ScPir4 over the entire amino acid sequence. The homology was higher $(65 \%)$ when only the C-terminal parts were compared (Fig. 1). Both C. albicans IPFs had almost the same amino acid sequence; the differences were that IPF 19968 had a gap of 39 aa and a point mutation $\left(\operatorname{Leu}_{281} / \mathrm{Ser}_{242}\right)$ (Fig. 1); this means that these two IPFs could be two alleles of the same gene. To investigate this, the promoter sequences of both IPFs were analysed. Comparison of the promoter sequences indicated that the two IPFs corresponded to the same gene. By analogy with ScPir4, the C. albicans gene was named CaPIR1.

\section{Structural analysis of the amino acid sequence encoded by CaPIR1}

CaPIR1 encodes two putative polypeptides, of 385 aa (IPF 15363 ) and 364 aa (IPF 19968), respectively, with calculated molecular masses of 40.5 and $36.4 \mathrm{kDa}$ and pIs of 4.85 and $4 \cdot 75$. Analysis of the predicted amino acid sequences revealed an $\mathrm{N}$-terminal region with characteristics of a signal peptide (von Heijne, 1986) and a predicted cleavage site at positions 18-19 (...SLA-AT...). Assuming that the cleavage site is at position 18, the mature proteins consisted of 367 aa and 328 aa, respectively, with a calculated molecular mass of $38.9 \mathrm{kDa}$ (IPF 15363 ) and $34 \cdot 7 \mathrm{kDa}$ (IPF 19968). Approximately $20 \%$ of the amino acid residues are Ser or Thr in both IPFs, indicating that CaPirl could be a highly $O$ glycosylated protein. One potential $\mathrm{N}$-glycosylation site (N-Xaa-S/T) was identified at amino acid position 233-235 (NST) in IPF 15363 and 194-196 (NST) in IPF 19968. Another structural feature of CaPir1 is the presence of four Cys residues in the $\mathrm{C}$-terminal part of the protein (-C-66aaC-16aa-C-12aa-C-COOH) (Fig. 1), which is characteristic of the ScPir protein family. ScPir proteins are also identified by the presence of amino acid repeats in their sequences; in this regard ScPir4 has one [-(S-Q-(I/V)-(S/T/G/N/H)-D-G$\mathrm{Q}-(\mathrm{L} / \mathrm{I} / \mathrm{V})-\mathrm{Q}-(\mathrm{A} / \mathrm{I} / \mathrm{V})-(\mathrm{S} / \mathrm{T} / \mathrm{A})-]$ whereas CaPir1 has nine (IPF 15363) or seven (IPF 19968) with a slightly different structure [-(A/K/Q)-Q-I-(S/T/G/N)-D-G-Q-I-Q-H-Q-T-].

\section{Expression patterns of CaPIR1}

The presence of different alleles of CaPIR1 opened the possibility of finding if both alleles were expressed equally under the same environmental conditions or if they had a different expression control. The expression of CaPIR1 was examined by RT-PCR. Fig. 2(a, b) shows the RTPCR amplification of CaPIR1-specific fragments (1120 and $1003 \mathrm{bp}$ ) from first-strand cDNA derived from cells growing in different conditions. Different samples, containing the same quantity of first-strand cDNA, were prepared and subjected to different cycles of amplification. Fig. 2(a, b) shows that the quantity of CaRPSO-specific amplicon (545 bp) was approximately the same in each of the firststrand cDNA samples. The presence of one intron in the corresponding region of the CaRPSO genomic DNA allows differentiation between bands amplified from cDNA and any contaminating genomic DNA (870 bp) (Baquero et al., 2001). The results indicated that the two alleles of CaPIR1 were expressed in the same amount independently of the cell morphology, yeast or mycelium (Fig. 2a). In $S$. cerevisiae at least one of the Pir proteins is a heat-shock protein (Hsp150/Pir2; Kapteyn et al., 1999a); to determine if CaPirl could be a heat-shock protein and so a functional homologue of ScPir2, a semi-quantitative RT-PCR was performed from cells growing at $25^{\circ} \mathrm{C}$ and then transferred to $37^{\circ} \mathrm{C}$. As shown in Fig. 2(b), no differences in expression were observed, suggesting that CaPir1 is not a heat-shock 


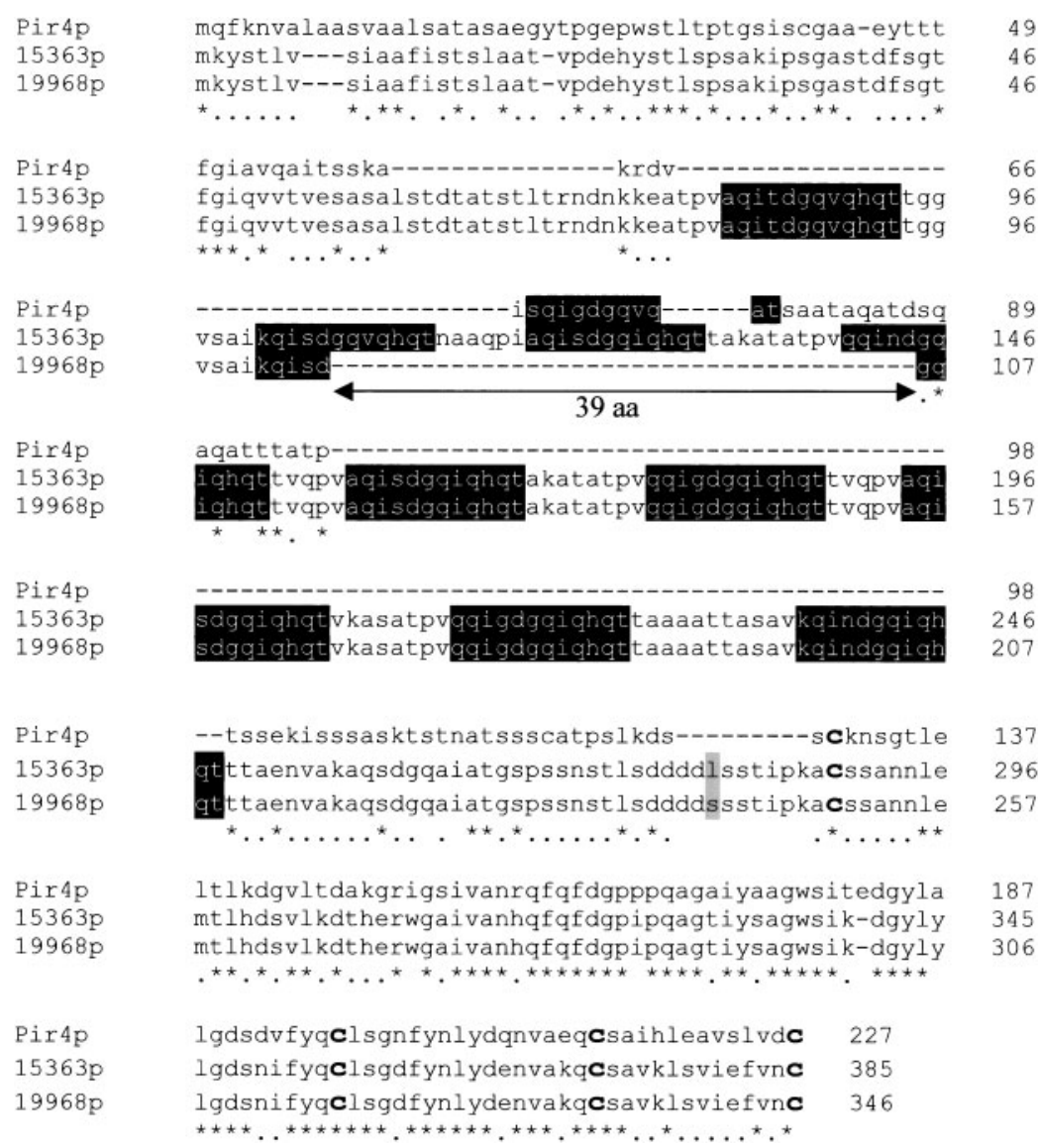

Fig. 1. Alignment of the S. cerevisiae Pir4 sequence and the sequences deduced from IPFs 19968 and 15363. Internal repetitions are shadowed in black, the point mutation $\left(\mathrm{Leu}_{281} / \mathrm{Ser}_{242}\right)$ in grey and the four conserved cysteine residues in bold. The arrow indicates the 39 aa lacking in the sequence deduced from IPF 19968. protein. One interesting observation was made from protoplasts under cell wall regeneration conditions. C. albicans protoplasts were incubated in a regeneration medium, and after 30, 60, 120, 180 and $300 \mathrm{~min}$, samples were taken and the CaPIR1 expression examined by RT-PCR. The results (Fig. 2c) indicated that CaPIR1 expression increased with time of regeneration, showing a maximum after $120 \mathrm{~min}$ of incubation.

\section{Construction of CaPIR1 mutants}

To investigate the function of CaPir1 protein, construction of null mutants by targeted gene disruption was attempted. Disruption of the CaPIR1 gene was performed by using a strategy originally developed for S. cerevisiae (Alani et al., 1987) and modified for use in C. albicans (Fonzi \& Irwin, 1993). This method uses a cassette consisting of the C. albicans URA3 gene flanked by direct repeats of the Salmonella typhimurium hisG gene. This cassette was used to replace approximately $50 \%$ of the ORF of the two alleles of CaPIR1 (IPF 15363 and IPF 19968). A linear SacI-PstI fragment from plasmid p15-c, including the cassette flanked by CaPIR1 sequences, was used to transform C. albicans CAI4 to $\mathrm{Ura}^{+}$(see Methods).

Fourteen of the resulting $\mathrm{Ura}^{+}$transformants were analysed and eleven of them contained the desired insert at the
CaPIR1 locus (data not shown). Southern blot analysis of different isolates, after digestion with SacI, revealed that the cassette had integrated into one of the two CaPIR1 alleles (Fig. 3a), giving rise to a $13 \cdot 8 \mathrm{~kb}$ fragment; this is consistent with the replacement of one allele of CaPIR1 with the transforming DNA. The $10 \mathrm{~kb}$ SacI fragment corresponds to the other allele which was still present in the Ura ${ }^{+}$transformants. To determine which of the two alleles had been disrupted, an RT-PCR assay was performed. As shown in Fig. 3(b), disruptants of the two alleles were obtained. A representative isolate of each mutant was chosen; these isolates were named $C$. albicans CAPIR15 and C. albicans CAPIR19, heterozygous mutants for allele IPF 15363 and IPF 19968, respectively. Ura ${ }^{-}$segregants were selected on medium containing 5-fluoro-orotic acid (Boeke et al., 1984) and examined by Southern blot analysis. More than 60 independent segregants were examined and all of them had experienced an interchromosomal recombination event, reverting to the C. albicans CAI4 genotype (data not shown). By this method of disruption only heterozygous mutants in one or other of the two CaPIR1 alleles were obtained (Fig. 3b) so we tried to obtain null mutants by the technique of Wilson et al. (1999). By this technique again only heterozygous mutants were obtained (data not shown). By using this technique it is not necessary to obtain any $\mathrm{Ura}^{-}$ segregants to disrupt the second allele, and the fact that no homozygous mutants were obtained with both techniques 


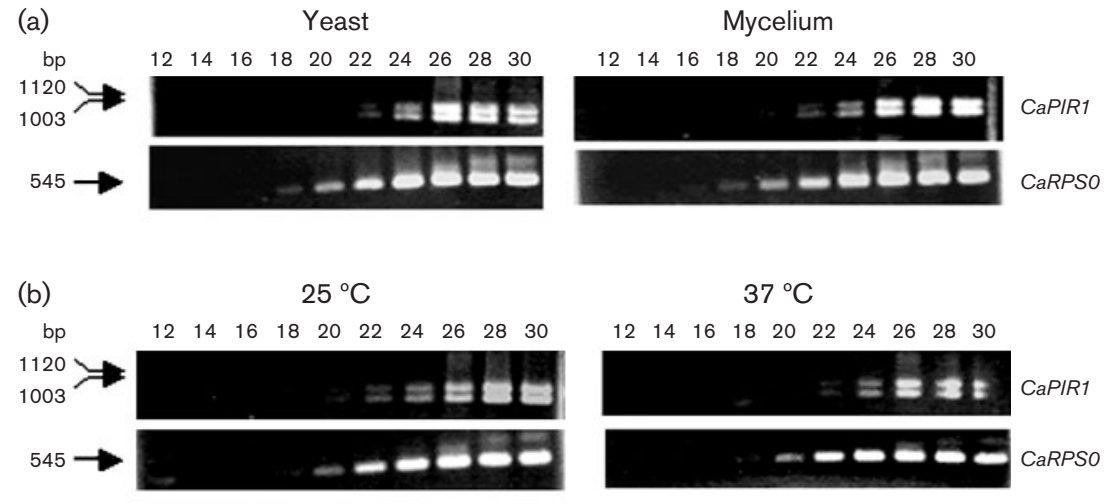

(c)

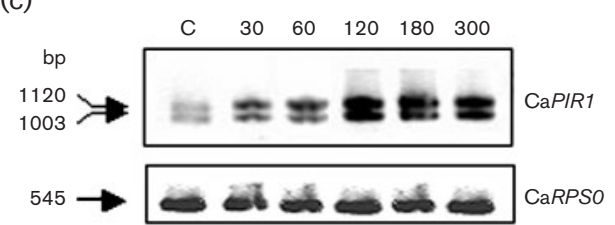

Fig. 2. (a, b) Expression of CaPIR1 mRNA under different conditions measured by semiquantitative RT-PCR: (a) yeast and mycelium; (b) heat shock (25 and $37^{\circ} \mathrm{C}$ ). Samples containing $1 \mu \mathrm{g}$ cDNA were amplified and several cycles of amplification were analysed (cycle numbers are shown at the top of each panel). (c) Protoplast regeneration at $28^{\circ} \mathrm{C}(30,60,120,180$ and $300 \mathrm{~min}$ regeneration) measured by RTPCR, using as control cDNA obtained from an exponential-phase CAI4 culture in YNB medium at $28^{\circ} \mathrm{C}(\mathrm{C})$. Samples containing $1 \mu \mathrm{g} \mathrm{cDNA}$ were subjected to 30 cycles of amplification and then run on a $0.8 \%$ agarose gel. Primers that amplified a $545 \mathrm{bp}$ fragment of CaRPSO (a constitutive gene with a intron in its sequence) were used as control. Primers that amplified the complete ORF of CaPIR1 were used to measure CaPIR1 expression. could indicate that CaPIR1 is an essential gene for cell viability.

\section{Phenotypic analysis of the Capir1/CaPIR1 mutants}

Phenotypic analysis of the heterozygous mutants obtained in comparison with the parental strain was performed. The specific growth rates of the cells at $28^{\circ} \mathrm{C}$ and $37^{\circ} \mathrm{C}$ on minimal medium were slower in both heterozygous mutants than in the parental strain (data not shown). In addition, cells of C. albicans CAPIR15 and CAPIR19 mutant strains showed some differences in morphology when they were grown at $28^{\circ} \mathrm{C}$ or $37^{\circ} \mathrm{C}$ (Fig. 4a). Mutant cells appeared elongated and many of them had an abnormal shape with a tendency to form clumps of different numbers of cells (Fig. 4a).
Possible alterations in the cell wall were studied by testing the sensitivities of the two heterozygous strains, C. albicans CAPIR15 and CAPIR19, to Calcofluor white and Congo red, as described in Methods. Sensitivities to these compounds did increase in the two mutant strains with respect to C. albicans CAI4 parental cells (Fig. 4b), suggesting that both CaPIR1 alleles, IPF 15363 and IPF 19968, contribute to maintaining the architecture of the cell wall.

\section{Overexpression and cellular localization of CaPir1 in C. albicans}

Overexpression of CaPIR1 was achieved by subcloning an amplicon containing the IPF 15363 allele of CaPIR1 in a pADH episomal vector (Bertram et al., 1996) under the control of the $A D H 1$ promoter. The new plasmid was named pADH-15 and it was used to transform C. albicans CAI4. To (a)

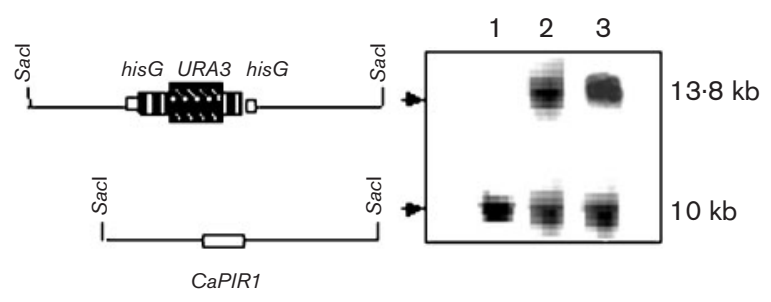

(b)

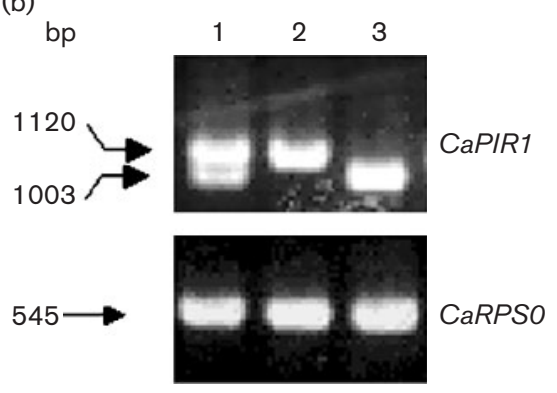

Fig. 3. (a) Southern blot analysis of genomic DNAs from C. albicans CAI4, CAPIR15 and CAPIR19 digested with Sacl and hybridized with a DIG-labelled DNA probe (amplicons 15-1,2 and 15-3,4). The wild-type and disrupted alleles are shown on the left. (b) RT-PCR analysis of the transformants using ADH $155^{\prime}$ and ADH $153^{\prime}$ as primers. Primers that amplified a 545 bp fragment of CaRPSO (a constitutive gene with a intron in its sequence) were used as control. Primers that amplified the complete ORF of CaPIR1 were used to measure CaPIR1 expression. 
(a)

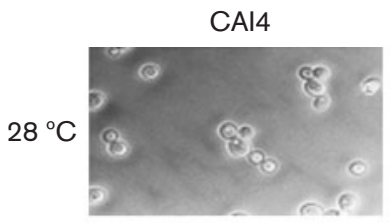

$37^{\circ}$

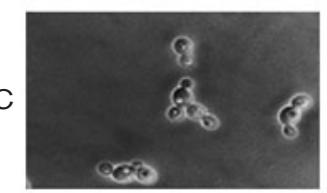

CAPIR15
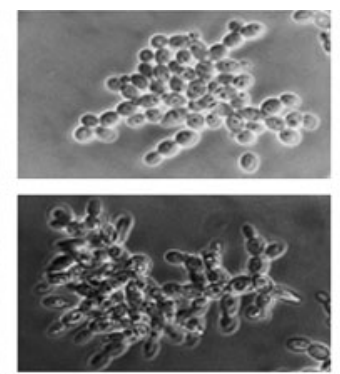

(b)

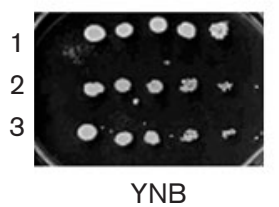

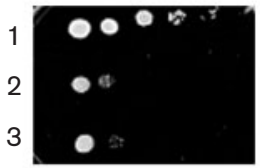

CFW $\left(100 \mu \mathrm{g} \mathrm{ml}^{-1}\right)$

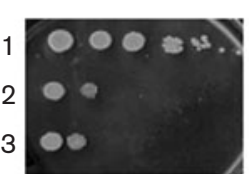

$\mathrm{CR}\left(10 \mu \mathrm{g} \mathrm{ml^{-1 } )}\right.$

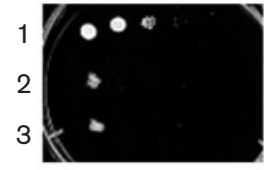

CFW $\left(150 \mu \mathrm{g} \mathrm{ml}^{-1}\right)$

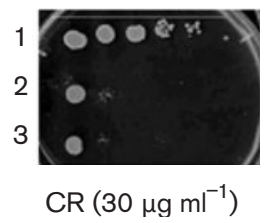

Fig. 4. (a) Morphology of C. albicans strains CAl4 and CAPIR15 in YNB medium at $28^{\circ} \mathrm{C}$ and $37^{\circ} \mathrm{C}$. (b) Sensitivities to Calcofluor white (CFW) and Congo red (CR) of the parental (1) and heterozygous strains (CAPIR15 and CAPIR19; 2 and 3 respectively). Cells were grown in YPD medium and a 1/10 dilution series of each strain was inoculated on to YNB medium containing the indicated amounts of CR and CFW.

determine in which cell wall fraction the overexpressed material was located, a Western blot analysis of $\beta$ mercaptoethanol and alkaline cell wall extracts, and also the spent medium, using PAbL antibodies, was performed. An increased amount of the material released by alkaline solutions, but not in the material released by $\beta$-mercaptoethanol or present in the spent medium, was observed in the strain that overexpressed CaPIR1 when compared with C. albicans CAI4 (Fig. 5a). No change in morphology or growth rate of cells was observed in the overexpressing C. albicans strain.

To confirm that the overexpressed material, detected in the alkaline extracts, corresponded to CaPirl, and not to other kinds of material recognized by the PAbL antibody, the allele IPF 15363 was tagged with the V5 epitope. This epitope is a 14 aa peptide (Gly-Lys-Pro-Ile-Pro-Asn-Pro-Leu-Leu-GlyLeu-Asp-Ser-Thr) that has been described as being convenient for tagging C. albicans extracellular proteins (Spreghini et al., 2003). The V5 epitope was introduced into the C-terminal end of CaPirl as described in Methods. As shown in Fig. 5(b), the recombinant protein was bound directly to the $1,3-\beta$-glucan, as it could only be released from the cell wall by treatment with alkaline solutions. Small amounts of the protein were also detected in the spent medium (Fig. 5b).

The difference between the predicted size of CaPir1 (about $40 \mathrm{kDa}$ in the case of the allele IPF 15363) and that deduced from the mobility in SDS-PAGE (about $180 \mathrm{kDa}$, Fig. 5b) could be accounted for by $\mathrm{N}$ - and/or $\mathrm{O}$-glycosylation. To determine if CaPirl was modified postranslationally by $\mathrm{N}$ glycosylation, the material released to the spent medium by C. albicans expressing CaPir1 tagged with V5 was treated with Endo-F. Western blot analysis with anti-V5 antibodies showed that the $180 \mathrm{kDa}$ species disappeared after Endo-F treatment, and a new species of $110 \mathrm{kDa}$ appeared (Fig. 5c), indicating that CaPirl was $\mathrm{N}$-glycosylated. There is a substantial difference between the molecular mass of CaPir1 (IPF 15363) treated with Endo-F (110 kDa) as determined by SDS-PAGE, and its predicted molecular mass $(40 \cdot 5 \mathrm{kDa})$ calculated from the deduced amino acid sequence. This discrepancy could be accounted for by the potential $O$ glycosylation, as $20 \%$ of its amino acids are Ser/Thr, which is known to increase the apparent size in the Laemmli gel system.

\section{Expression of CaPIR1 in S. cerevisiae}

To analyse whether CaPirl could be incorporated into the walls of $S$. cerevisiae, cells of $S$. cerevisiae pir $4 \Delta$ transformed with plasmid pADH-15 were grown and their cell walls isolated. Cell walls were treated with $\beta$-mercaptoethanol or alkaline solutions; the spent medium was also analysed. The solubilized material was analysed by Western blotting using PAbL antibodies and ConA-peroxidase. A main band with an apparent molecular mass of $110 \mathrm{kDa}$ was detected in $\beta$ mercaptoethanol extracts (Fig. 6a). A band with a similar mobility was also detected in the spent medium and in the material extracted by alkaline solutions from the isolated cell walls of the S. cerevisiae CaPIR1-expressing strain (data not shown). No changes in either morphology or growth rate of cells were observed in S. cerevisiae pir $4 \Delta$ expressing CaPIR1.

As indicated above, CaPirl is $\mathrm{N}$-glycosylated in C. albicans. To determine whether the protein is also $\mathrm{N}$-glycosylated when expressed in $S$. cerevisiae, material obtained by $\beta$-mercaptoethanol was treated with Endo-F. No new species appeared (Fig. 6b), indicating that CaPir1 is not $\mathrm{N}$-glycosylated by the enzymic machinery of $\mathrm{S}$. cerevisiae.

\section{DISCUSSION}

Wall biogenesis in fungal cells, and specifically in C. albicans, is the result of several steps that are initiated by the synthesis of the proteins at the level of the endoplamic reticulum, followed by their glycosylation in the vesicles of the secretory pathway and release to the periplasmic space, where they interact with the nascent wall polysaccharides. The process ends with bond formation between the different components, producing the final architecture of the wall. Alhough 


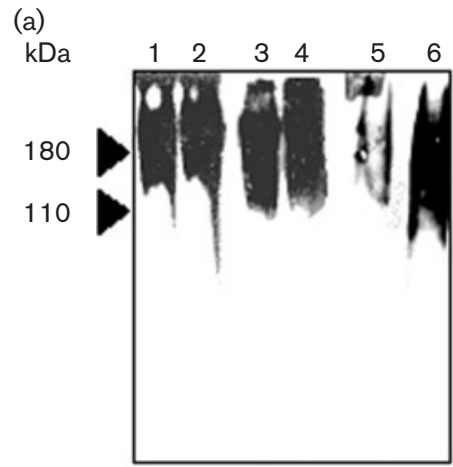

PAbL (b)

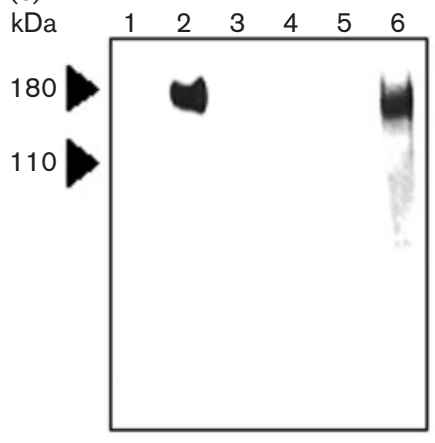

(c)

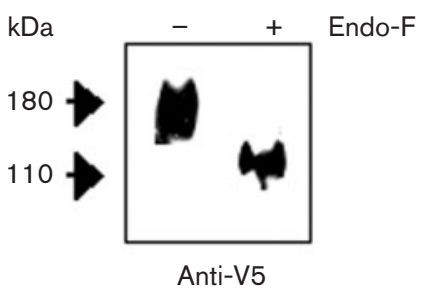

Anti-V5

Fig. 5. (a) Western blot analysis of the materials released to the culture medium (lanes 1 and 2), or extracted from purified cell walls by $\beta$-mercaptoethanol (lanes 3 and 4 ) or alkali solutions (lanes 5 and 6) from strains CAl4 (lanes 1, 3 and 5 ) and CAl4/pADH-15 (lanes 2, 4 and 6) reacted with PAbL polyclonal antibody against the cell wall of C. albicans in yeast morphology. (b) Western blot analysis of the material released to the culture medium (lanes 1 and 2), or extracted from purified cell walls by $\beta$-mercaptoethanol (lanes 3 and 4 ) or alkali solutions (lanes 5 and 6) from strains CAl4 and CAI4/pMAL15-V5 grown in YNB-maltose medium, probed with anti-V5 antibody. (c) Western blot analysis of the material released to the culture medium by strain CAI4/pMAL-15-V5 and developed with anti-V5 monoclonal antibody, before (-) and after (+) Endo$\mathrm{F}$ treatment.

some of the proteins and protein-polysaccharide bonds are fairly well known, identification of additional wall proteins and the enzymes catalysing formation of the different bonds is needed.

S. cerevisiae Pir4 was identified from the material released from isolated walls with $\beta$-mercaptoethanol (Castillo et al., 2003). New potential C. albicans cell wall proteins related to S. cerevisiae Pir4 were identified by an in silico search of a C. albicans database. Only two ORFs were found (IPF 15363 and 19968; Fig. 1). Additional BLAST searches in the
C. albicans database for other homologues of the Pir family of S. cerevisiae (Pir1, Pir2 and Pir3) gave negative results (Toh-e et al., 1993). Due to the similarity in the homology percentages, CaPirl can not be designated as the functional homologue of a specific Pir protein.

Three main differences have been detected in the C. albicans proteins in comparison to ScPir4: (i) absence of a potential Kex2 site (Lys-Arg, Arg-Arg and Pro-Arg) close to the Nterminal part of the proteins (cleavages occur at the carboxyl side of pairs of basic residues: Brenner et al., 1994; Mizuno

(a)
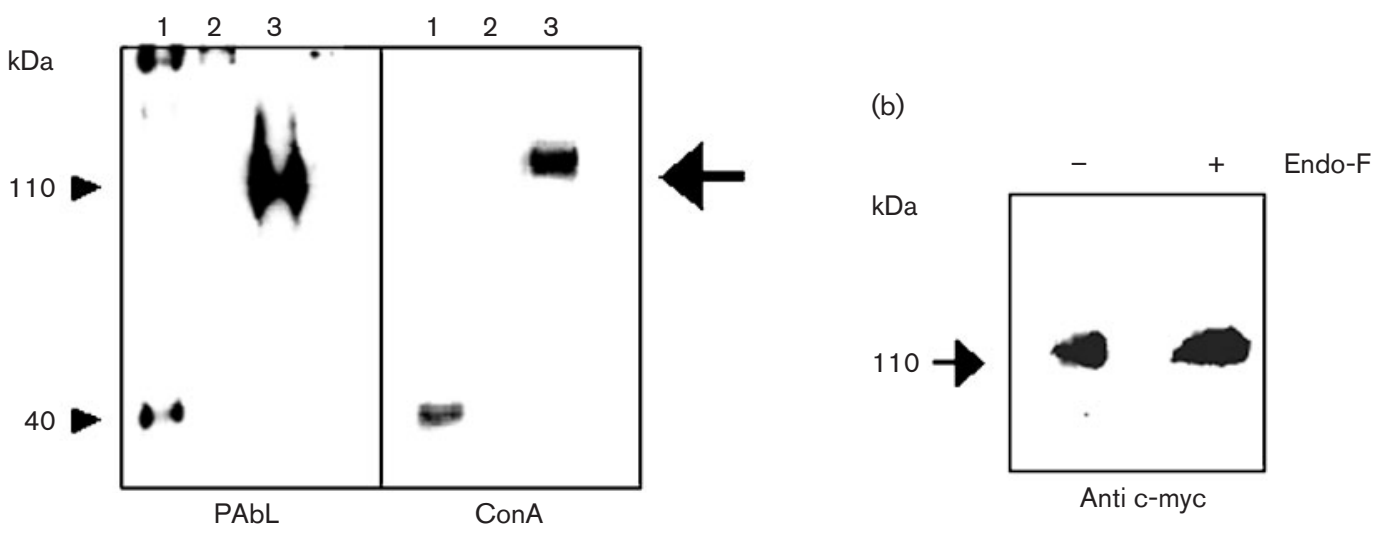

Fig. 6. (a) Analysis by SDS-PAGE and Western blotting of the materials released by $\beta$-mercaptoethanol from purified cell walls. The $S$. cerevisiae strains used were: 1 , BMA64-1A; 2 pir4 $\Delta ; 3$, pir4 $/ \mathrm{pADH}-15$. The nitrocellulose membranes were probed with PAbL and ConA-peroxidase. (b) Western blot analysis of $\beta$-mercaptoethanol extracts from purified cell walls of Scpir $4 \Delta / \mathrm{pADH}-15-\mathrm{myc}$ before $(-)$ and after $(+)$ Endo-F treatment, reacted with anti-c-myc monoclonal antibody. The $110 \mathrm{kDa}$ band is arrowed. 
et al., 1989; Goller et al., 1998); (ii) the C. albicans protein encoded by IPF 15363 has nine tandem repeats of 11 aa (QITDGQVQHQT) and IPF 19968 seven tandem repeats, whereas the ScPir4 has only one repeat (SQIGDGQVQA); and (iii) a point mutation $\left(\mathrm{Leu}_{281} / \mathrm{Ser}_{242}\right)$ that could be a real point mutation. A homologue of S. cerevisiae Hsp150/ Pir2 has been reported in C. albicans (Kapteyn et al., 2000; Kandasamy et al., 2000) but the results obtained in silico and by mass spectrometry (unpublished results) indicated that the product of a single PIR gene (CaPIR1) is found in C. albicans cell wall. Therefore it is possible that the protein previously reported is the same as the one described in this paper which we have named CaPirl.

It is interesting that both IPF 15363 and IPF 19968 are expressed under normal laboratory growth conditions, after heat shock and in both yeast and mycelial forms. The codon bias index (CBI) for IPF 15363 is $0 \cdot 345$, suggesting that it is a poorly expressed gene. The low expression of CaPIR1 contrasts with the situation of the PIR genes in S. cerevisiae because PIR1, PIR2 and PIR3 are among those expressed abundantly (Toh-e et al., 1993).

To define the cellular function of PIR1, we attempted to contstruct a null mutant to search for informative phenotypes. However, we were unable to obtain a null mutant even though both the Fonzi \& Irwin (1993) and Wilson et al. (1999) techniques were used. We have no explanation for these results, but the phenotype of the heterozygous mutants, independently of the allele interrupted (specific growth rates, clump formation and hypersensitivity to cellwall-perturbing agents such as Calcofluor white and Congo red) (Elorza et al., 1983; Ram et al., 1994; Mrša et al., 1999), and the fact that in S. cerevisiae there are four PIR genes instead of one, indicated the possibility that the null mutant is lethal. In the case of $S$. cerevisiae, sequential disruption of PIR genes brings increasingly irregular shape, clumping of the cells and a pronounced destabilization in the presence of Calcofluor white and Congo red (Mrša \& Tanner, 1999); therefore C. albicans PIR1 may be an essential gene.

The haploinsufficiency phenotypes indicate that both PIR1 alleles contribute to maintaining the correct cell wall organization in wild-type strains. This situation is in some respects similar to that in S. cerevisiae, as the phenotype shown by this species is progressively more apparent as the number of PIR genes disrupted increases (Mrša \& Tanner, 1999).

CaPir1 was found as a new cell wall band when expressed in S. cerevisiae. This band reacted with ConA, demonstrating that it was a glycoprotein, but it could not be detected in C. albicans as the material released from the wall was highly polydisperse and no specific antibodies were available. A recombinant CaPir1 tagged with the V5 epitope was found linked only to the $1,3-\beta$-glucan through an alkali-sensitive bond. MALDI-TOF analysis of the materials released by $\beta$ mercaptoethanol also failed to detect CaPir1 (unpublished). Therefore it is possible that CaPirl is only attached to the
1,3 - $\beta$-glucan of the wall. This result is surprising, as a fraction of S. cerevisiae Pir4 molecules and CaPir1 expressed in this species are retained in the wall by disulphide bridges and as a consequence released by reducing agents.

Nothing is known about the function of CaPir1. Toh-e et al. (1993) isolated in S. cerevisiae three highly homologous genes of the PIR family (PIR1, PIR2 and PIR3), and genes homologous to PIR have been also found in Kluyveromyces lactis and Zygosaccharomyces rouxii but not in Schizosaccharomyces pombe, suggesting that the PIR genes play a role in budding yeast. In addition, null mutants of each gene are viable, indicating that none of them is essential, but they are required for tolerance to heat shock (PIR2/HSP150) (Toh-e et al., 1993) and determine resistance to the plant protein osmotin (Yun et al., 1997; Ibeas et al., 2001). By functional genomics it has been found that ScPir4 interacts with Yjr030 and Bur2 (Ito et al., 2001) and it was suggested that it might strengthen the regenerating cell wall of protoplasts (Pardo et al., 1999). In this context it is important to emphasize that the S. cerevisiae Pir family is formed by four proteins (Pir1, Pir2, Pir3 and Pir4) whereas in C. albicans only one Pir homologue has been found. BLAST searches to find other members in the C. albicans genomic database have given negative results. Recently it has been reported that ScPir2 is more efficiently retained in the wall of $S$. cerevisiae growing at low $\mathrm{pH}$ and it was suggested that this is also the case for other Pir proteins (Kapteyn et al., 2001) and that probably all members of this protein family are at least functionally equivalent in the cell wall.

Although the actual function of CaPir1 is unknown, it may be critical in the organization of the wall because during the initial steps of protoplast regeneration $(2-3 \mathrm{~h})$ the levels of expression are significantly high.

Two interesting differences have been detected between CaPirl and ScPir4 from the structural and functional points of view: (i) ScPir4 is retained in the wall by two types of bonds (disulphide bridges and covalently bound to the 1,3$\beta$-glucan) whereas CaPir1 seems to be only attached to the 1,3 - $\beta$-glucan (the protein is not detected in the material extracted by $\beta$-mercaptoethanol by immunological or mass spectrometry techniques; unpublished observations); and (ii) four proteins are found in the S. cerevisiae Pir family whereas in C. albicans only one member of this family (CaPir1, as determined in silico) has been found. CaPir1 has an $\mathrm{N}$-glycosylation sequon in its sequence $\left(\mathrm{NX}_{\mathrm{aa}} \mathrm{S} / \mathrm{T}\right)$ at positions 233-235 and the protein moves with an apparent molecular mass of $180 \mathrm{kDa}$ that is reduced to $110 \mathrm{kDa}$ after treatment with Endo-F. But it seems that it is not $\mathrm{N}$ glycosylated when expressed by S. cerevisiae. This result is of interest but its reason is unknown; it could be due to the different specificity of the oligosaccharyltransferase (the enzyme complex that transfers the inner core of the carbohydrate moiety) in the two organisms, or to steric hindrance as occurs with $S$. cerevisiae invertase; this enzyme contains 14 sequons but only eight or nine are glycosylated (Reddy et al., 1988). 
Finally, the cell wall of $S$. cerevisiae cells has been engineered using Pir1 or Pir2 to anchor proteins by fusion of the corresponding genes with PIR1 or PIR2 and the enzymic activities produced by fusion proteins on their surface detected (Abe et al., 2004), opening the possibility to express C. albicans heterologous proteins by fusion of the corresponding genes with CaPIR1.

\section{ACKNOWLEDGEMENTS}

This work was partially supported by grants from the European Union (MRTN-CT-2003-504148), the Spanish Ministerio de Ciencia y Tecnología (BMC2003-01023) and Agència Valenciana de Ciència i Tecnologia de la Generalitat Valenciana (Grupos 03/187). We thank Ismail Moukadiri and Jesús Zueco for S. cerevisiae BMA64-1Apir4A.

\section{REFERENCES}

Abe, H., Ohba, M., Shimma, Y. \& Jigami, Y. (2004). Yeast cells harboring human alpha-1,3-fucosyltransferase at the cell surface engineered using Pir, a cell wall-anchored protein. FEMS Yeast Res 4, 417-425.

Alani, E., Cao, L. \& Kleckner, N. (1987). A method for gene disruption that allows repeated use of URA3 selection in the construction of multiply disrupted yeast strains. Genetics 116, 541-545.

Backen, A. C., Broadbent, I. D., Fetherston, R. W., Rosamond, J. D. C., Schnell, N. F. \& Stark, M. J. R. (2000). Evaluation of the CaMAL2 promoter for regulated expression of genes in Candida albicans. Yeast 16, 1121-1129.

Baquero, C., Montero, M., Sentandreu, R. \& Valentín, E. (2001). Molecular cloning of the RPSO gene from Candida tropicalis. Yeast 18, 971-980.

Berman, J. \& Sudbery, P. E. (2002). Candida albicans: a molecular revolution built on lessons from budding yeast. Nat Rev Genet 12, 918-930.

Bertram, G., Swoboda, R. K., Gooday, G. W., Gow, N. A. \& Brown, A. J. (1996). Structure regulation of the Candida albicans $A D H 1$ gene encoding an immunogenic alcohol dehydrogenase. Yeast 12, 115-127.

Boeke, J. D., LaCroute, F. \& Fink, G. R. (1984). A positive selection for mutants lacking orotidine- $5^{\prime}$-phosphate decarboxylase activity in yeast: 5-fluoro-orotic acid resistance. Mol Gen Genet 197, 345-346.

Brenner, C., Bevan, A. \& Fuller, R. S. (1994). Biochemical and genetic methods for analyzing specificity and activity of a precursorprocessing enzyme: yeast Kex2 protease, kexin. Methods Enzymol 244, 152-167.

Burnette, W. N. (1981). "Western blotting": electrophoretic transfer of proteins from sodium dodecyl sulfate-polyacrylamide gels to unmodified nitrocellulose and radiographic detection with antibody and radioiodinated protein A. Anal Biochem 112, 195-203.

Calderone, R. A. \& Fonzi, W. A. (2001). Virulence factors of Candida albicans. Trends Microbiol 19, 327-335.

Castillo, L., Martínez, A. I., Garcerá, A., Elorza, M. V., Valentín, E. \& Sentandreu, R. (2003). Functional analysis of cysteine residues and the repetitive sequences of ScPir4: the first repetitive sequence is needed to binding the cell wall $\beta$-1,3-glucan. Yeast 20, 973-983.

Cleves, A. E., Cooper, D. N., Barondes, S. H. \& Kelly, R. B. (1996). A new pathway for protein export in Saccharomyces cerevisiae. J Cell Biol 133, 1017-1026.
Elorza, M. V., Rico, H. \& Sentandreu, R. (1983). Calcofluor white alters the assembly of chitin fibrils in Saccharomyces cerevisiae and Candida albicans cells. J Gen Microbiol 129, 1577-1582.

Elorza, M. V., Marcilla, A. \& Sentandreu, R. (1988). Wall mannoproteins of the yeast and mycelial cells of Candida albicans: nature of the glycosidic bonds and polydispersity of their mannan moieties. J Gen Microbiol 134, 2393-2403.

Eroles, P., Sentandreu, M., Elorza, M. V. \& Sentandreu, R. (1997). The highly immunogenic proteins enolase and Hsp70 are adventitious Candida albicans cell wall proteins that act as virulence factors. Microbiology 143, 313-320.

Fonzi, W. A. \& Irwin, M. Y. (1993). Isogenic strain construction and gene mapping in Candida albicans. Genetics 134, 717-728.

Gietz, R. D. \& Sugino, A. (1988). New yeast-Escherichia coli shuttle vectors constructed with in vitro mutagenized yeast genes lacking six-base pair restriction sites. Gene 74, 527-534.

Gietz, D., St Jean, A., Woods, R. A. \& Schiestl, R. H. (1992). Improved method for high efficiency transformation of intact yeast cells. Nucleic Acids Res 20, 1425.

Gillum, A. M., Tsay, E. Y. \& Kirsch, D. R. (1984). Isolation of the Candida albicans gene for orotidine- 5 '-phosphate decarboxylase by complementation of $S$. cerevisiae ura3 and E. coli pyrF mutations. Mol Gen Genet 198, 179-182.

Goller, S. P., Schoisswohl, D., Baron, M., Parriche, M. \& Kubicek, C. P. (1998). Role of endoproteolytic dibasic proprotein processing in maturation of secretory proteins in Trichoderma reesei. Appl Environ Microbiol 64, 3202-3208.

Hanahan, D. (1985). Techniques for transformation of Escherichia coli. In DNA Cloning: a Practical Approach, p. 109. Edited by D. M. Glover. Oxford: IRL Press.

Hawkes, R. (1982). Identification of concanavalin A-binding proteins after sodium dodecyl sulfate-gel electrophoresis and protein blotting. Anal Biochem 80, 348-355.

Ibeas, J. I., Yun, D. J., Damsz, B. \& 7 other authors (2001). Resistance to the plant PR-5 protein osmotin in the model fungus Saccharomyces cerevisiae is mediated by the regulatory effects of SSD1 on cell wall composition. Plant J 25, 271-280.

Ito, H., Fukuda, Y., Murata, K. \& Kimura, A. (1983). Transformation of intact yeast cells treated with alkali cations. J Bacteriol 153, 163-168.

Ito, T., Chiba, T., Ozawa, R., Yoshida, M., Hattori, M. \& Sakaki, Y. (2001). A comprehensive two-hybrid analysis to explore the yeast protein interactome. Proc Natl Acad Sci U S A 98, 4569-4574.

Jaafar, L., Moukadiri, I. \& Zueco, J. (2003). Characterization of a disulphide-bound Pir-cell wall protein (Pir-CWP) of Yarrowia lipolytica. Yeast 20, 417-426.

Kandasamy, R., Vediyappan, G. \& Chaffin, W. L. (2000). Evidence for the presence of Pir-like proteins in Candida albicans. FEMS Microbiol Lett 186, 239-243.

Kapteyn, J. C., Van Egmond, P., Van Den Ende, H., Makarow, M. \& Klis, F. M. (1999a). The contribution of the O-glycosylated protein Pir2p/Hsp150 to the construction of the yeast cell wall in wildtype cells and beta 1,6-glucan-deficient mutants. Mol Microbiol 31, 1835-1844.

Kapteyn, J. C., Van Den Ende, H. \& Klis, F. M. (1999b). The contribution of cell wall proteins to the organization of the yeast cell wall. Biochim Biophys Acta 1426, 373-383.

Kapteyn, J. C., Hoyer, L. L., Hecht, J. E., Muller, W. H., Andel, A., Verjleij, A. J., Makarow, M., Van Den Ende, H. \& Klis, F. M. (2000). The cell wall architecture of Candida albicans wild-type cells and cell wall-defective mutants. Mol Microbiol 35, 601-611. 
Kapteyn, J. C., ter Riet, B., Vink, E., Blad, S., De Nobel, H., Van Den Ende, H. \& Klis, F. M. (2001). Low external pH induces HOG1dependent changes in the organization of the Saccharomyces cerevisiae cell wall. Mol Microbiol 39, 469-479.

Klis, F. M., de Groot, P. \& Hellingwerf, K. (2001). Molecular organization of the cell wall of Candida albicans. Med Mycol 39, $1-8$.

Klis, F. M., Mol, P., Hellingwerf, K. \& Brul, S. (2002). Dynamics of cell wall structure in Saccharomyces cerevisiae. FEMS Microbiol Rev 3, 239-256.

Laemmli, U. K. (1970). Cleavage of structural proteins during the assembly of the head of bacteriophage T4. Nature 227, 680-685.

Langford, C. J. \& Gallwitz, D. (1983). Evidence for an introncontained sequence required for the splicing of yeast RNA polymerase II transcripts. Cell 33, 519-527.

Marcilla, A., Elorza, M. V., Mormeneo, S., Rico, H. \& Sentandreu, R. (1991). Candida albicans mycelial wall structure: supramolecular complexes released by zymolyase, chitinase and $\beta$-mercaptoethanol. Arch Microbiol 155, 312-319.

Marcilla, A., Mormeneo, S., Elorza, M. V., Manclus, J. J. \& Sentandreu, R. (1993). Wall formation by Candida albicans yeast cells: secretion and incorporation of two types of mannoproteins. J Gen Microbiol 139, 2985-2993.

Millete, C. F. \& Scott, B. K. (1984). Identification of spermatogenic cell plasma membrane glycoproteins by two dimensional electrophoresis and lectin blotting. J Cell Sci 65, 233-248.

Mizuno, K., Nakamura, T., Ohshima, T., Tanaka, S. \& Matsuo, H. (1989). Characterization of KEX2-encoded endopeptidase from yeast Saccharomyces cerevisiae. Biochem Biophys Res Commun 159, 305-311.

Mormeneo, S., Marcilla, A., Iranzo, M. \& Sentandreu, R. (1995). Structural mannoproteins released by $\beta$-elimination from Candida albicans cell walls. FEMS Microbiol Lett 123, 131-136.

Moukadiri, I., Jaafar, L. \& Zueco, J. (1999). Identification of two mannoproteins released from cell walls of a Saccharomyces cerevisiae mnn1 mnn9 double mutant by reducing agents. J Bacteriol 181, 4741-4745.

Mrša, V. \& Tanner, W. (1999). Role of $\mathrm{NaOH}$-extractable cell wall proteins Ccw5p, Ccw6p, Ccw7p and Ccw8p (members of the Pir protein family) in stability of the Saccharomyces cerevisiae cell wall. Yeast 15, 813-820.

Mrša, V., Ecker, M., Strahl-Bolsinger, S., Nimtz, M., Lehle, L. \& Tanner, W. (1999). Deletion of new covalently linked cell wall glycoproteins alters the electrophoretic mobility of phosphorylated wall components of Saccharomyces cerevisiae. J Bacteriol 181, 3076-3086.
Pardo, M., Monteoliva, L., Plá, J., Sánchez, M., Gil, C. \& Nombela, C. (1999). Two-dimensional analysis of proteins secreted by Saccharomyces cerevisiae regenerating protoplasts: a novel approach to study the cell wall. Yeast 15, 459-472.

Pardo, M., Ward, M., Bains, S., Molina, M., Blackstock, W., Gil, C. \& Nombela, C. (2000). A proteomic approach for the study of Saccharomyces cerevisiae cell wall biogenesis. Electrophoresis 21, 3396-3410.

Ram, A. F., Wolters, A., Ten Hoopen, R. \& Klis, F. M. (1994). A new approach for isolating cell wall mutants in Saccharomyces cerevisiae by screening for hypersensitivity to calcofluor white. Yeast 10, 1019-1030.

Reddy, V. A., Johnson, R. S., Biemann, K., Williams, R. S., Ziegler, F. D., Trimble, R. B. \& Maley, F. (1988). Characterization of the glycosylation sites in yeast external invertase. I. N-linked oligosaccharide content of the individual sequons. J Biol Chem 263, 6978-6985.

Sentandreu, R., Elorza, M. V. \& Ruiz-Herrera, J. (2001). Structure, synthesis and assembly of fungal cell wall glycoproteins. In Recent Research Developments in Microbiology, pp. 23-33. Edited by S. G. Pandalai. Trivandrum, India: Research Signpost.

Spreghini, E., Davis, D. A., Subaran, R., Kim, M. \& Mitchell, A. P. (2003). Roles of Candida albicans Dfg5p and Dcwlp cell surface proteins in growth and hypha formation. Eukaryot Cell 2, 746-755.

Sundstrom, P. (2002). Adhesion in Candida spp. Cell Microbiol 8, 461-469.

Toh-e, A., Yasunaga, S., Nisogi, H., Tanaka, K., Oguchi, T. \& Matsui, Y. (1993). Three yeast genes, PIR1, PIR2 and PIR3, containing internal tandem repeats, are related to each other, and PIR1 and PIR2 are required for tolerance to heat shock. Yeast $\mathbf{9}$, 481-494.

Towbin, H., Staehelin, T. \& Gordon, J. (1979). Electrophoretic transfer of proteins from polyacrylamide gels to nitrocellulose sheets: procedure and some applications. Proc Natl Acad Sci U S A 76, 4350-4354.

Van der Vaart, J. M., Caro, L. H., Chapman, J. W., Klis, F. M. \& Verrips, C. T. (1995). Identification of three mannoproteins in the cell wall of Saccharomyces cerevisiae. J Bacteriol 177, 3104-3110.

Von Heijne, G. (1986). New method for predicting signal sequence cleavage sites. Nucleic Acids Res 11, 4683-4690.

Wilson, R. B., Davis, D. \& Mitchell, A. P. (1999). Rapid hypothesis testing with Candida albicans through gene disruption with short homology regions. J Bacteriol 181, 1868-1874.

Yun, D. J., Zhao, Y., Pardo, J. M. \& 7 other authors (1997). Stress proteins on the yeast cell surface determine resistance to osmotin, a plant antifungal protein. Proc Natl Acad Sci U S A 94, 7082-7087. 